\title{
Ist die Verweigerung eines Internetzugangs im Strafvollzug rechtmäßig?
}

\begin{abstract}
Summary
The article asks whether the general denial of an internet connection in prison is legal. The starting point is the fact that the internet represents a basic societal change which was inconceivable when the federal penal code was implemented. Today, daily life without the internet is unimaginable for many people. With the federalism reform, the legislative competency in matters of penal law was transferred to the federal states; therefore, at that time there was an opportunity to examine the issue of internet and the prison system in the context of law-making. While considering the states' laws which were passed on the prison system and the sample draft, the article looks into the question posed above from the following perspectives: The penal system as rule enforcement, the penal system's goal of avoiding "adverse effects," and the principle of assimilation in the context of contact to the outside world. The author comes to the conclusion that the denial of internet access in the penal system can be legal if it is absolutely necessary to maintain security or to avoid a serious disruption of the institution's order. However, abstract danger cannot be given as a reason, as this would be equivalent to a denial. In summary, it can be said that the general denial of internet access is illegal. The problem of internet access within the prison system is alluded to only in the sample draft that includes regulations on "devices for information and entertainment technology, " which can be permitted under certain conditions.
\end{abstract}

\section{Résumé}

L'article examine dans quelle mesure le refus de principe d'accorder un accès internet à des détenus est légal ou non. On part du principe qu'internet a provoqué une profonde transformation sociale, que l'on ne pouvait pas prévoir au moment de la mise en application de la loi sur l'exécution des peines par l'Etat fédéral. Or, internet est aujourd'hui devenu indissociable de la vie de nombreuses personnes. Le transfert de l'exécution des peines dans le domaine de compétence des Etats fédérés du fait de la réforme du système fédéral aurait pu offrir la possibilité de s'intéresser à la question d'internet et de l'exécution des peines dans le cadre du droit de légiférer ainsi accordé. En se référant aux lois concernant l'exécution des peines promulguées par les Länder et au projet-cadre, l'article étudie la question de départ sous les angles de vue suivants: l'exécution de la peine pour les récidivistes, qu'en est-il de l'objectif visant à éviter les " influences néfastes » sur les détenus, et la question de l'harmonisation des comportements du fait du contact avec le monde extérieur. L'auteur en arrive finalement à la conclusion que refuser l'accès internet en détention peut être légal, si ceci est indispensable pour assurer la sécurité ou pour éviter de graves perturbations au règlement de la prison. Il ne convient toutefois pas de se considérer, de manière abstraite, internet 
comme un danger, car cela reviendrait à en refuser par principe l'accès aux détenus. On doit donc en conclure qu'il n'est pas légal de refuser, de manière générale, la connexion à internet. Le problème de la connexion internet en détention n'a été abordé à mots couverts que dans le projet-cadre, qui contient des dispositions touchant aux « moyens électroniques de l'information et de distraction » ne pouvant être autorisés que sous certaines conditions.

Die zu untersuchende Frage, ob eine Verweigerung eines Anspruchs auf Internetzugang im Strafvollzug rechtmäßig ist, geht von der These aus, dass sich unsere Gesellschaft rasant verändert und das Internet aus dem Alltag der Menschen nicht mehr wegzudenken ist (Onlinebanking, Online-Stellensuche, Onlineshopping, Informationen, Soziale Netzwerke).

Das Thema wirft neue Fragen auf, weil zum einen zum Zeitpunkt des Inkrafttretens des StVollzG am 1.1.1977 das Internet im Alltagsgebrauch noch nicht verankert war und weil es zum anderen Strafgefangene gab und gibt, die in einer Industriegesellschaft inhaftiert wurden und nun in eine Informationsgesellschaft entlassen werden. Selbst Menschen, die schon in Zeiten der Informationsgesellschaft inhaftiert wurden, werden aufgrund der rasanten Entwicklung im Bereich Digitalisierung der Gesellschaft abgehängt und in einen für sie unbekannten Zustand entlassen. Wer heute den Strafvollzug verlässt, wird sich in wichtigen Lebensbereichen nicht mehr zurechtfinden. Die Chancen auf Resozialisierung sinken, die Chancen auf dem Arbeitsmarkt sowieso.

Die rechtlichen Ausführungen begegnen der Schwierigkeit, dass mit der Föderalismusreform die Zuständigkeit für die Gesetzgebung auf dem Gebiet des Strafvollzuges auf die Länder übergegangen ist. Ein Musterentwurf für Landesgesetze liegt ebenso vor und die Länder Bayern, Baden-Württemberg, Hamburg, Hessen und Niedersachsen haben bereits von der Gesetzgebungskompetenz Gebrauch gemacht. Umfangreiche juristische Ausarbeitungen gibt es aber lediglich zum StVollzG (Bund). Die bisher getroffenen Regelungen und ebenso der Musterentwurf weichen von der Formulierung in $\S 2$ StVollzG (Bund) ab.

Letztendlich gilt: „Eine allgemeine Aussage über den Sinn des Strafvollzuges oder seine Ziele und Zwecke berührt das religiöse und weltanschauliche Verständnis des Betroffenen und der Allgemeinheit über Schuld, Verantwortung und Sühne."1

\section{Offener Vollzug als Regelvollzug, Freiheitsstrafe als Einschränkung der Bewegungsfreiheit}

Der Theorie und dem bisherigen StVollzG (Bund) nach müsste der offene Vollzug in Deutschland der Regelvollzug sein. In $\S 10$ Abs. 1 StVollzG heißt es: „Ein Gefangener soll mit seiner Zustimmung in eine Anstalt oder Abteilung des offenen Vollzuges untergebracht werden, wenn er den besonderen Anforderungen des offenen Vollzuges genügt und namentlich nicht zu befürchten ist, dass er sich dem Vollzug der Freiheitsstrafe entziehen oder die Möglichkeiten des offenen Vollzuges zu Straftaten missbrau-

1 Bundestagsdrucksache 7/918, S. 44. 
chen werde.“ Der $\S 10$ Abs. 2 lautet: „Im Übrigen [sic!] sind die Gefangenen im geschlossenen Vollzug unterzubringen. [...].“ Diese Auslegung des $§ 10$ StVollzG wird durch die Gesetzesmaterialien zum StVollzG gestützt, in denen es heißt: „...dass die mit einer geschlossenen Anstalt verbundene Isolierung nur dann vertreten werden kann, wenn der Gefangene sich sonst dem Vollzug der Freiheitsstrafe entziehen oder andere, lockere Formen der Beaufsichtigung und Unterbringung zu weiteren Straftaten missbrauchen würde. In einer Anstalt des geschlossenen Vollzuges sollen deshalb nur solche Gefangene untergebracht werden, die nicht im offenen Vollzug untergebracht werden können oder die dies wünschen“. ${ }^{2}$ Der Musterentwurf hält sich im Wesentlichen an den Normgehalt des $\S 10$ StVollzG (Bund). Auch Baden-Württemberg und Niedersachsen lassen eine Anlehnung an den Wesensgehalt des $\S 10$ StVollzG (Bund) erkennen. Bayern und Hessen normieren eine Regelunterbringung im geschlossenen Vollzug, auch Hamburg schränkt den Wesensgehalt des bisherigen $\S 10$ StVollzG (Bund) ein. Das Bundesverfassungsgericht dürfte wohl zur Auslegung des offenen Vollzuges als Regelvollzug tendieren, wenn es formuliert: ,Soweit keine Flucht- oder Missbrauchsgefahr besteht, ist er für geeignete Gefangene die Regelvollzugsform und nicht eine besondere Vergünstigung." 3

Zur Einordnung ist es notwendig zu klären, was unter Freiheitsstrafe zu verstehen ist. Soll die Freiheitsstrafe lediglich die Bewegungsfreiheit einschränken oder folgt aus ihrer Verhängung, dass die Gefangenen weitere Einschränkungen hinzunehmen haben? Christoph Flügge beantwortet die Frage im Sinne einer Einschränkung lediglich der Bewegungsfreiheit, wenn er schreibt: „Nun noch einige Worte zum Gefängnissystem in Deutschland. In Deutschland ist die Freiheitsstrafe eine einheitliche Freiheitsstrafe, die auf den Entzug der persönlichen Bewegungsfreiheit gerichtet ist. Sonstige Belastungen der Gefangenen sind nicht legitimiert, § 4 Abs. 2 StVollzG."4 Auch bei Roggan und Kutscha heißt es: „Nur wirklich schwere Rechtsverstöße oder andere Belange erheblichen Gewichts sollen den Entzug der Bewegungsfreiheit zur Konsequenz haben. Dabei bestimmt das Gesetz selber, dass kurze Freiheitsstrafen nur in Ausnahmefällen verhängt werden dürfen ( $\$ 47 \mathrm{StGB}) . " 5$ Gleiches dürfte sich auch aus Artikel $104 \mathrm{GG}$ ergeben. Absatz 1 lautet: „Die Freiheit der Person kann nur auf Grund eines förmlichen Gesetzes und nur unter Beachtung der darin vorgeschriebenen Formen beschränkt werden. Festgehaltene Personen dürfen weder seelisch noch körperlich mißhandelt werden.” Bei Dannecker im Leipziger Kommentar zum StGB heißt es diesbezüglich: „Diese Verfassungsvorschrift schützt die körperliche Bewegungsfreiheit vor Verhaftung, Festnahme und ähnlichen Eingriffen, also vor unmittelbarem Zwang. Deshalb ist, wenn Freiheitsstrafe angedroht wird, gemäß Art. 104 Abs. 1 S. 1 GG stets ein förmliches Gesetz (oder eine ihm gleichstehende gesetzesvertretende Verordnung, Rdn. 125) erforderlich." "Auch bei Beckemper findet sich eine Formulierung, die Freiheitsstrafe als Entzug der Bewegungsfreiheit definiert: „Vielmehr ergebe sich der ultima ratio-Cha-

2 Bundestagsdrucksache 7/918, S. 51 und Bundesratsdrucksache 71/73, S. 49.

3 BVerfG, 2 BvR 725/07, 27.9.2007.

4 http://www.juridicainternational.eu/public/pdf/ji_2003_1_43.pdf, zuletzt abgerufen am 15.6.2012.

5 Roggan/Kutscha, Handbuch zum Recht der Inneren Sicherheit, S. 247.

6 Dannecker, Leipziger Kommentar zum StGB, § 1, Rdn. 123. 
rakter des Strafrechts aus der Besonderheit der 'Freiheitsstrafe', die mit der Bewegungsfreiheit den Kernbereich der bürgerlichen Freiheit betreffe."7

Es bleibt mithin festzustellen, dass die Freiheitsstrafe den Entzug der Bewegungsfreiheit meint und entgegen der Praxis der offene Vollzug der Regelvollzug ist.

\section{Vollzugsgrundsatz ,schädliche Auswirkungen“vermeiden}

Bereits im Jahr 1972 hat das Bundesverfassungsgericht festgestellt: „Auch die Grundrechte von Strafgefangenen können nur durch Gesetz oder aufgrund eines Gesetzes eingeschränkt werden. [...] Eine Einschränkung der Grundrechte des Strafgefangenen kommt nur in Betracht, wenn sie zur Errichtung eines von der Werteordnung des Grundgesetzes gedeckten gemeinschaftsbezogenen Zweckes unerlässlich ist."

Bereits im sog. Lebach-Urteil hat das Bundesverfassungsgericht dem Ziel der Resozialisierung verfassungsrechtliche Bedeutung beigemessen. Dort heißt es: „Als maßgebender Orientierungspunkt für die nähere Bestimmung der zeitlichen Grenze kommt das Interesse an der Wiedereingliederung des Straftäters in die Gesellschaft, an seiner Resozialisierung in Betracht. Die Erkenntnis, welche Bedeutung diese Zielsetzung hat, setzte sich in den letzten Jahrzehnten im Strafrecht zunehmend durch; nach allgemeiner Auffassung wird die Resozialisierung oder Sozialisation als das herausragende Ziel namentlich des Vollzuges von Freiheitsstrafen angesehen (vgl. auch BVerfGE 33, 1; http:// www.servat.unibe.ch/dfr/bv033001.html" \1 "007). Dem Gefangenen sollen Fähigkeit und Willen zu verantwortlicher Lebensführung vermittelt werden, er soll es lernen, sich unter den Bedingungen einer freien Gesellschaft ohne Rechtsbruch zu behaupten, ihre Chancen wahrzunehmen und ihre Risiken zu bestehen." 9 Das Bundesverfassungsgericht hat in seiner Entscheidung vom 26.10. 2011 wiederholt bestätigt, dass das Grundrecht aus Art. 2 Abs. 1 i.V.m. Art. 1 Abs. 1 GG den Staat verpflichtet, den Strafvollzug auf das Ziel auszurichten, dem Inhaftierten eine zukünftiges straffreies Leben in Freiheit zu ermöglichen. Es hat darüber hinaus kürzlich darauf verwiesen, dass im Hinblick auf Art. 2 GG in Verbindung mit Art. 1 GG die Vollzugsanstalten verpflichtet sind, ,schädlichen Auswirkungen des Strafvollzuges im Rahmen des Möglichen zu begegnen"10

Das StVollzG (Bund) legt in $§ 2$ Satz 1 bereits als Vollzugsziel fest, dass der Gefangene im Vollzug der Freiheitsstrafe fähig werden soll, zukünftig in sozialer Verantwortung ein Leben ohne Straftaten zu führen. Bei einem Blick auf den Musterentwurf wie auch auf die Landesgesetze zeichnet sich hier wohl aber ein Prioritätenwechsel ab. Der Musterentwurf formuliert in $\S 2$,Der Vollzug dient dem Ziel, die Gefangenen zu befähigen, künftig in sozialer Verantwortung ein Leben ohne Straftaten zu führen. Er hat die Aufgabe, die Allgemeinheit vor weiteren Straftaten zu schützen. “Im Gegensatz zum $\S 2$ Satz 2 StVollzG (Bund) fehlt hier das Wörtchen ,auch“. ${ }^{11}$ Buch $1 \S 2$ in Baden-

7 Beckemper, ZStW 2006, S. 259ff.

8 BVerfGE 33, 1 (BVerfG, 14.3.1972, 2 BvR 41/71).

9 BVerfGE 35, S. 202, Rdn. 70.

10 siehe BVerfG, 2 BvR 729/08, 5.8.2010.

11 „Der Vollzug der Freiheitsstrafe dient auch dem Schutz der Allgemeinheit vor weiteren Straftaten.". 
Württemberg sieht die Aufgabe im Schutz der Bürgerinnen und Bürger vor weiteren Straftaten und erst in Satz zwei darin, einen Beitrag für u.a. die Wiedereingliederung zu leisten. ${ }^{12}$ In Buch $3 \S 1$ wird dann als Vollzugsziel definiert, dass der Gefangene im Vollzug fähig werden soll, künftig in sozialer Verantwortung ein Leben ohne Straftaten zu führen. Bayern nennt in Artikel 2 als Vollzugsziel zunächst in Satz eins den Schutz der Allgemeinheit vor weiteren Straftaten und in Satz zwei die Befähigung, künftig in sozialer Verantwortung ein Leben ohne Straftaten zu führen. ${ }^{13}$ Hamburg wiederum nennt in $\S 2$ zunächst die Resozialisierung als Vollzugsziel, stellt aber durch das Wort „gleichermaßen“14 klar, dass gleichberechtigt auch der Schutz der Allgemeinheit vor weiteren Straftaten zum Vollzugsziel gehört. In Hessen wird in § 2 Satz drei klargestellt, dass sowohl die Resozialisierung als auch die sichere Unterbringung dem Schutz der Allgemeinheit vor weiteren Straftaten dient. ${ }^{15}$ Niedersachsen wiederum stellt durch das Wort ,zugleich“ in $\S 5^{16}$ ebenfalls die Gleichrangigkeit der Vollzugsziele sicher. Inwiefern sowohl der Musterentwurf als auch die Landesgesetze dem vom Bundesverfassungsgericht vorgegebenen Ziel gerecht werden, kann und soll hier nicht weiter untersucht werden.

Auch in der Rechtswissenschaft hat sich der Gedanke der Resozialisierung als herrschende Meinung im Hinblick auf das Vollzugsziel durchgesetzt. Bei Köhne heißt es prägnant: „Die Resozialisierung stellt das einzige Ziel des Strafvollzuges dar. Die Resozialisierung dient dem Schutz der Gesellschaft, da sie verhindern soll, dass der Täter rückfällig wird und erneut die Gemeinschaft schädigt. Allein die erfolgreiche soziale (Wieder-)Eingliederung des Verurteilten in die Gesellschaft wird dem Sicherheitsbedürfnis der Öffentlichkeit langfristig gerecht. Die Vollzugsaufgabe des Schutzes der Allgemeinheit vor weiteren Straftaten ( $\$ 2$ S. 2 StVollzG) ist dem Vollzugsziel der Resozialisierung daher nachgeordnet bzw. sogar am besten durch dieses zu lösen. ${ }^{17}$

Diesem Vollzugsziel entsprechend werden sowohl im StVollzG (Bund) als auch im Musterentwurf und den Landesgesetzen Präzisierungen vorgenommen. Der sogenannte Angleichungsgrundsatz findet sich in allen - wenn auch in unterschiedlicher Wortwahl und um andere Aspekte ergänzt - wieder. Der $\S 3$ StVollzG (Bund) schreibt vor, dass das Leben im Vollzug den allgemeinen Lebensverhältnissen soweit als möglich angeglichen werden soll, schädlichen Folgen des Freiheitsentzuges entgegenzuwirken ist und der Vollzug darauf ausgerichtet sein soll, dem Gefangenen zu helfen, sich in das Leben in Freiheit einzugliedern.

In der Begründung des Gesetzesentwurfes des Bundesrates heißt es dazu: „Die Entwurfsvorschrift begründet deshalb die Verpflichtung der Vollzugsbehörden, Besonderheiten des Anstaltslebens, die den Gefangenen lebensuntüchtig machen können, zurückzudrängen, so dass der Unterschied zwischen dem Leben in der Anstalt und dem Leben draußen nicht stärker als unvermeidbar ist. Dieser Grundsatz wirkt sich auf das

12 vgl. http://www.jva-adelsheim.de/servlet/PB/show/1247938/jvad_Justizvollzugsgesetz-buc h.pdf, zuletzt abgerufen am 15.6.2012.

13 vgl. http://www.justizvollzug-bayern.de/JV/stvollzg2008.pdf, zuletzt abgerufen am 15.6.2012.

14 vgl. http://www.justizvollzug-bayern.de (Fn. 13).

15 vgl. http://www.justizvollzug-bayern.de (Fn. 13).

16 vgl. http://www.recht-niedersachsen.de/34210/njvollzg.htm, zuletzt abgerufen am 15.6.2012.

17 Köhne, Resozialisierungsunfähige Strafgefangene, ZRP 6/2003, S. 207. 
gesamte System des Vollzuges aus, ist bei allen einzelnen Maßnahmen gebührend zu berücksichtigen, $[\ldots] .{ }^{18}$

Feest/Lesting vertreten die Position, dass der Angleichungsgrundsatz den weiteren Gestaltungsgrundsätzen vorgeht. Gleichzeitig sollen im Falle einer Regelungslücke die für alle Bürger geltenden Normen und Gesetze auf die Gefangenen Anwendung finden. ${ }^{19}$

\section{Angleichungsgrundsatz in Bezug zum Kontakt mit der Außenwelt}

Zum Zeitpunkt des Inkrafttretens des Strafvollzugsgesetzes gab es das Internet im alltäglichen Leben der Menschen noch nicht. Ebenso wenig das Mobiltelefon. Heute ist das Internet aus dem Alltag der Menschen nicht mehr wegzudenken. Mit Freunden und Bekannten wird Kontakt über soziale Netzwerke gehalten, offene Arbeitsstellen und Stellengesuche sind online im Angebot, Banküberweisungen erfolgen online und unzensierte und ungefilterte Originalinformationen finden sich ebenfalls online.

Eine Analogie zu anderen im StVollzG (Bund) geregelten Materien hilft hier nicht weiter, da diese nicht an die Bedeutung des Internets für die Gestaltung und Bewältigung des Alltags heranreichen. Buch $1 \S 22$ des Gesetzes zur Umsetzung der Föderalismusreform im Bereich des Justizvollzuges Baden-Württemberg untersagt explizit die Nutzung von Mobilfunkgeräten. ${ }^{20}$ Dem gegenüber formuliert der $\S 36$ des Musterentwurfes: „Nach Zulassung anderer Formen der Telekommunikation im Sinne des Telekommunikationsgesetzes durch die Aufsichtsbehörde kann der Anstaltsleiter den Gefangenen gestatten, diese Formen auf ihre Kosten zu nutzen. Die Bestimmungen dieses Abschnitts gelten entsprechend." Und in $\S 51$ Rundfunk, Informations- und Unterhaltungselektronik: ,(1) Der Zugang zum Rundfunk ist zu ermöglichen. (2) Eigene Hörfunk- und Fernsehgeräte werden zugelassen, wenn nicht Gründe des $\S 48$ Satz 2 entgegenstehen. Andere Geräte der Informations- und Unterhaltungselektronik können unter diesen Voraussetzungen zugelassen werden. Die Gefangenen können auf Mietgeräte oder auf ein Haftraummediensystem verwiesen werden."

Der Musterentwurf scheint zumindest das Problem erkannt zu haben und nicht von einer völligen Untersagung auszugehen. Das StVollzG (Bund) kennt also keine Regelung, die es grundsätzlich ausschließt, Geräte der Informations- und Unterhaltungselektronik zu nutzen. Der Musterentwurf selbst normiert dies sogar als Möglichkeit.

\section{Zugang zum Internet für Strafgefangene}

Das Bundesverfassungsgericht hat im Jahr 1972 ausgeführt: „In Artikel 1 Abs. 3 GG werden die Grundrechte für Gesetzgebung, vollziehende Gewalt und Rechtsprechung für unmittelbar verbindlich erklärt. Dieser umfassenden Bindung widerspräche es, wenn im Strafvollzug die Grundrechte beliebig oder nach Ermessen eingeschränkt werden

18 Bundesratsdrucksache 71/73, S. 44 und Bundestagsdrucksache 7/918, S. 46.

19 vgl. Feest/Lesting, StVollzG, § 3, Rdn. 4, 6.

20 vgl. http://www.jva-adelsheim.de (Fn. 12). 
könnten. Eine Einschränkung kommt nur dann in Betracht, wenn sie zur Erreichung eines von der Werteordnung des Grundgesetzes gedeckten gemeinschaftsbezogenen Zweckes unerlässlich ist und in den dafür vorgesehenen Formen geschieht. Die Grundrechte von Strafgefangenen können also nur durch Gesetz oder aufgrund eines Gesetzes eingeschränkt werden..."21 Die Frage, ob die Verweigerung eines Internetzugangs für Strafgefangene rechtswidrig ist, soll nach den bisherigen Ausführungen unter folgenden Prämissen untersucht werden:

- Freiheitsstrafe nach dem StGB meint den Entzug der Bewegungsfreiheit.

- Schädliche Auswirkungen des Freiheitsentzuges sind auch darin zu sehen, dass entlassene Strafgefangene sich in einer digitalisierten Welt nicht zu Recht finden.

- Der Strafvollzug hat im Rahmen des Möglichen schädlichen Auswirkungen der Haft zu begegnen.

- Entsprechend $\S 3$ StVollzG (Bund), den Regelungen in den Ländern und des Musterentwurfs soll das Leben im Vollzug den allgemeinen Lebensverhältnissen soweit als möglich angeglichen werden.

Soweit nicht der Auffassung von Feest/Lesting gefolgt wird, dass im Falle einer fehlenden Norm die Normen und Gesetze gelten, denen alle Bürger/innen unterworfen sind, kann unter diesen Prämissen die Verweigerung eines Internetzugangs für Strafgefangene allein dann rechtmäßig sein, wenn sie für die Aufrechterhaltung der Sicherheit oder zur Abwendung einer schwerwiegenden Störung der Ordnung der Anstalt unerlässlich ist bzw. unter den im Musterentwurf angegebenen Bedingungen. Da der $\S 51$ Abs. 2 des Musterentwurfes auf den $\S 48$ Satz 2 Musterentwurf ${ }^{22}$ verweist, ist auch insoweit auf die Sicherheit und Ordnung der Anstalt bzw. die Übersichtlichkeit des Haftraums abzustellen. Die Erreichung des Vollzugsziels als Ausschlusskriterium wird in den weiteren Ausführungen ausgeschlossen.

\section{Gefahr für Sicherheit und Ordnung der Anstalt}

Sicherheit und Ordnung der Anstalt sind unbestimmte Rechtsbegriffe und insoweit gerichtlich nachprüfbar. Es verwundert also nicht, dass die wesentlichen Aussagen, was erlaubt und was verboten ist, durch Gerichte entschieden worden sind. Allerdings formulierte der Gesetzentwurf der Bundesregierung wie folgt: „... wobei der Begriff der Sicherheit der Anstalt sowohl die Abwendung von Gefahren für Personen und Sachen in der Anstalt als auch die Sicherung des durch den Freiheitsentzug begründeten Gewahrsams umfasst.“

Feest/Boetticher weisen zur Recht darauf hin, dass der Begriff der „Gefährdung“ ein Wahrscheinlichkeitsurteil enthält und weisen darüber hinaus darauf hin, dass bei Auslegung und Anwendung ,unter Inkaufnahme von Risiken der gesetzliche Vorrang der Wiedereingliederung vor Sicherheitserwägungen“ $\mathrm{zu}$ beachten ist. „Insbesondere der Angleichungsgrundsatz ( $§ 3$ Abs. 1) führt bei der Ausgestaltung des Vollzuges zu einer engen Auslegung der Begriffe Sicherung und Ordnung. Die mit den Begriffen Sicherheit

21 BVerfGE 33, 1 ff., 11.

22 "Gegenstände, die geeignet sind, die Sicherheit oder Ordnung der Anstalt, insbesondere die Übersichtlichkeit des Haftraumes, oder die Erreichung des Vollzugsziels zu gefährden, dürfen nicht in den Haftraum eingebracht werden oder werden daraus entfernt.“. 
bzw. Ordnung verbundene Prognoseunsicherheit muss zu einer Risikoverteilung zwischen Anstalt und Gefangenen führen, statt einseitig Rechtsbeschränkungen der Strafgefangenen zu legitimieren. Verbleibende Restrisiken müssen im Hinblick auf die Prinzipien des $\S 3$ und den Verhältnismäßigkeitsgrundsatz hingenommen werden. “23

Dieser Sicht ist der Vorzug zu geben vor der sehr einengenden Sicht von Kruis/ Wehofsky, die formulieren: ,So ist es von Verfassung wegen zwar unbedenklich, bereits die einem Gegenstand generell innewohnende Gefährlichkeit zum Ausgangspunkt einer Besitzversagung im Rahmen von $\S 70$ Abs. 1 und $\S 70$ Abs. 2 StVollzG zu nehmen, da es sich nicht um einen Eingriff in ein Freiheitsrecht, sondern um eine vom Gesetzgeber festgelegte Inhaltsbestimmung eines Rechts handelt. Allerdings ist die generell-abstrakte Gefährlichkeit in Beziehung zu setzen zu den Kontrollmöglichkeiten, insbesondere dem Einsatz milderer Mittel, etwa durch Verplombung technischer Geräte (Grundsatz der Erforderlichkeit der Maßnahme) sowie im Rahmen der Prüfung der Zumutbarkeit (Verhältnismäßigkeit im engeren Sinne) zu wichtigen Belangen des Gefangenen." 24

In Zeiten, in denen das Internet noch keine Alltagserscheinung war, wurde auf die generell innewohnende Gefährlichkeit abgestellt und postuliert, diese reiche zur Besitzversagung aus. Wer diese generelle Gefährlichkeit feststellt, wird nicht gesagt, ebenso wenig, was die Kriterien für eine solche Einordnung sein könnten. Wie es scheint, hat der Musterentwurf hier Konsequenzen gezogen und sich von der These der abstrakten Gefährlichkeit als Versagungsgrund abgesetzt. Wird der Sicht von Feest/ Boetticher gefolgt, kann mit dem Argument einer generell innewohnenden Gefährlichkeit des Internetzugangs die Verweigerung desselben nicht begründet werden. Wobei die Gefährlichkeit auch erst einmal begründet werden müsste. Daran dürfte auch die Entscheidung des Bundesverfassungsgerichtes vom 31. März 2003 nichts ändern, in der es um den Besitz eines Laptops für einen Strafgefangenen geht. Darin hieß es: „Nach $\S 70$ Abs. 2 Nr. 2 StVollzG besteht dieses Recht unter anderem dann nicht, wenn der Besitz, die Überlassung oder die Benutzung des Gegenstands die Sicherheit oder Ordnung der Anstalt gefährden würde. Das Vorliegen einer solchen Gefährdung kann ohne Verfassungsverstoß allein aufgrund der grundsätzlich gegebenen Eignung eines Gegenstandes für sicherheits- oder ordnungsgefährdende Verwendungen bejaht werden, sofern konkrete derartige Verwendungen nur mit einem von der Anstalt nicht erwartbaren Kontrollaufwand ausgeschlossen werden könnten (s. Beschlüsse der 2. Kammer des Zweiten Senats des Bundesverfassungsgerichts vom 28. Februar 1994 - 2 BvR 2731/93 -, NStZ 1994, S. 453 und vom 24. März 1996 - 2 BvR 222/96 -, NStZ-RR 1996, S. 252 sowie vom 12. Juni 2002 - 2 BvR 697/02 -)." ${ }^{25}$ Sowohl die Verweise auf frühere Entscheidungen als auch die Entscheidungsgründe lassen erkennen, dass die Frage eines Internetzugangs nicht Gegenstand der Abwägungen waren. Es ging in der Entscheidung um die Speicherfähigkeit eines Laptops, nicht aber um den Internetzugang.

Soweit ersichtlich hat sich die Rechtsprechung wohl weitgehend der These der generell innewohnenden Gefährlichkeit angeschlossen, wenn es um Geräte geht, mit denen

23 Feest/Boetticher, StVollzG, § 70, Rdn. 13.

24 Kruis/Wehofsky, Fortschreibung der verfassungsrechtlichen Leitsätze zum Vollzug von Strafund Untersuchungshaft, NStZ 1998, S. 594.

25 http://www.bundesverfassungsgericht.de/entscheidungen/rk20030331_2bvr184802.html, zuletzt abgerufen am 15.6.2012. 
Daten ausgetauscht werden können und der Zugang zum Internet möglich ist. Unter Verweis auf die Sicherheit und Ordnung der Anstalt hat das Landgericht Hildesheim beispielsweise entschieden: ,Jede - stets veränderbare - Datei müsste im Rahmen einer Kontrolle aufgerufen und gelesen werden. Verzichten könnte die Antragsgegnerin auf eine solche Kontrolle nicht. Es besteht stets die Gefahr des Abspeicherns - eventuell auch kodierter - Dateien mit sicherheitsgefährdenden Inhalten, etwa Ausbruchsplänen. Dass einem Gefangenen ein Zugriff auf das Internet und damit entgegen $\S \S 28$ Abs. 2 , 29 Abs. 3, 31 StVollzG die Möglichkeit zur unkontrollierten Kommunikation mit Außenstehenden und zu den teilweise strafbaren Inhalten mancher Internetseiten nicht gestattet werden muss, bedarf ohnehin keiner näheren Erörterung. "26 Auch das Brandenburgische Oberlandesgericht hat in einer internetfähigen Spielkonsole eine ,generellabstrakte Gefährlichkeit für die Sicherheit der Justizvollzugsanstalt“ gesehen und formuliert: „Der unkontrollierte Zugriff von Strafgefangenen auf das Internet würde den Austausch von Nachrichten und die Beschaffung von Informationen auf eine Weise ermöglichen, die mit den Sicherheitsbedürfnissen einer Justizvollzugsanstalt, [...] nicht zu vereinbaren sind". 27

Die Entscheidungen der Gerichte verdienen ebenso Widerspruch, wie die These der generell innewohnenden Gefährlichkeit. Eine solche These nämlich unterstellt, dass eigentlich dem Internet eine generelle Gefährlichkeit innewohnt. Tatsächlich lassen sich sowohl über Schreiben an und von Gefangenen als auch über Besuche all jene Dinge machen, die einen Internetzugang angeblich so viel gefährlicher erscheinen lassen. Niemand würde aber auf die Idee kommen, im Grundsatz jeglichen Schriftverkehr und jeglichen Besuch für Strafgefangene zu verbieten.

Die Gerichtsentscheidungen dürften auch im Gegensatz zum vom Bundesrat verfolgten Ziel stehen. Im Gesetzentwurf des Bundesrates zum StVollzG nämlich heißt es: „Die Entwurfsvorschrift verpflichtet deshalb die Vollzugsbehörden, die gesamte Vollzugstätigkeit auf eine wirkungsvolle, dem genannten Ziel dienende Behandlung des Gefangenen auszurichten. Einzelne Teilaspekte wie etwa die Arbeit des Gefangenen oder sein sicherer Gewahrsam sollen nicht als voranging behandelt werden dürfen. [...] Der strafweise Eingriff, den die Freiheitsstrafe auch als Behandlung darstellt, soll nur soweit eine Grundlage für Beschränkungen des Betroffenen darstellen, wie es für die Verbrechensverhütung und die Bekämpfung der Kriminalität notwendig ist." 28

$\mathrm{Zu}$ keinem anderen Ergebnis kommt man, wenn man sich auf den ehemaligen Justizminister Jahn bezieht, der sagte: „Zum anderen gilt es zu begreifen, dass die Sozialisation nicht erreicht werden kann durch weitgehende Isolation. Es war und ist falsch, den Gefangenen in eine Welt zu versetzen, in der er jede Beziehung zur Wirklichkeit des täglichen Lebens verlieren muss. Die Zerstörung der Verbindungen zur Umwelt, die über das vom Vollzug bestimmte, unvermeidliche Maß hinausgeht, ist Unsinn. Wie soll sich denn der ehemalige Gefangene in der Gesellschaft behaupten, wenn er sich in ihr nicht mehr auskennt?"29

26 LG Hildesheim, 4.5.2006, $23 \mathrm{StVK}$ 45/06.

27 BrbOLG, 26.1.2007, 2 Ws (Vollz) 103/06.

28 Gesetzentwurf des Bundesrates, Drucksache 71/73, S. 43 und Bundestagsdrucksache 7/918, S. 45.

29 Jahn, Rede im Bundestag am 19. Oktober 1973, Bundestagesplenarprotokoll 7/58, S. 3367. 
Vor diesem Hintergrund kann die abstrakte Gefährlichkeit kein Grund sein, einen Internetzugang zu versagen. Dies dürfte sich insoweit auch aus dem Musterentwurf ergeben

\section{Sicherheit und Ordnung der Anstalt im Verhältnis zum Vollzugsziel}

Die Argumentation der Gefahr für die Sicherheit und Ordnung der Anstalt allein kann also nicht die Versagung eines Internetzugangs begründen. Es muss demnach geprüft werden, ob im Rahmen der Verhältnismäßigkeit im engeren Sinne die Versagung des Internetzugangs erfolgen kann.

Analog entsprechender Entscheidungen des Bundesverfassungsgerichts zum Zugang zu Zeitschriften könnte eine Verweigerung des Zugangs wohl dann begründet sein, wenn der Zugang das Vollzugsziel gefährden würde. Ob dies bei Straftaten, die mittels des Internets begangen werden, der Fall ist, müsste im Einzelfall konkret geprüft werden.

Soweit dem Bundesverfassungsgericht gefolgt wird, wenn es formuliert: „Der Gefangene soll so lebenstüchtig bleiben, dass er sich im Falle einer Entlassung aus der Haft im normalen Leben wieder zurechtfindet, " 30 und soweit akzeptiert wird, dass das Internet eine immer bedeutendere Rolle im Leben und damit im Alltag der Menschen spielt, kann auch im Rahmen der Verhältnismäßigkeitsprüfung die generelle Verweigerung eines Zugangs zum Internet nicht als rechtmäßig angesehen werden. Es muss nämlich davon ausgegangen werden, dass ohne die Möglichkeit des Zugangs zum Internet gerade kein „Zurechtfinden im normalen Leben“ nach der Entlassung aus dem Strafvollzug möglich wäre. Genau hier liegt der zentrale Unterschied zu Fernseher, Spielkonsole etc., welche der Freizeitbeschäftigung dienten. Der Zugang zum Internet geht darüber hinaus, er ist notwendig, um sich Fähigkeiten und Fertigkeiten anzueignen, die für das Leben außerhalb des Strafvollzuges unerlässlich sind.

Soweit argumentiert wird, dass mittels des Internets beispielsweise Ausbruchpläne etc. ausgetauscht werden könnten und eine Überwachung wie im Schriftverkehr nicht möglich ist, ist dies nicht von der Hand zu weisen. Selbstverständlich wäre dies möglich. Allerdings muss hier eine Abwägung zwischen dem Vollzugsziel Resozialisierung und der dem Internet tatsächlich innewohnenden Gefahr des Informationsaustausches auch über Ausbruchspläne getroffen werden. Diese Abwägung kann vor dem Hintergrund des Vollzugsziels Resozialisierung aber nicht dazu führen, dass prinzipiell der Zugang zum Internet verweigert werden darf. Das Vollzugsziel hat als die ranghöchste Aufgabe beim Vollzug der Freiheitsstrafe zu gelten und andere Vollzugsaufgaben haben im Zweifel zurückzutreten. ${ }^{31}$

Unter Berücksichtigung des Angleichungsgrundsatzes in $\S 3$ Abs. 1 ist die Verweigerung eines Internetzugangs somit auch nicht mit der Gefährdung der Sicherheit und Ordnung der Anstalt zu begründen. Bei Feest/Lesting heißt es: „Die außerhalb der Anstalten üblichen Informations- und Kommunikationsmittel sind auch innerhalb der Anstalt zuzulassen. “32

30 BVerfG, 26.10.2011, 2 BvR 1539/09.

31 vgl. Feest/Lesting, StVollzG, § 2, Rdn. 5.

32 Feest/Lesting, StVollzG, §3, Rdn. 15. 


\section{Ergebnis und Handlungsbedarf des Gesetzgebers}

Eine generelle Verweigerung des Zugangs zum Internet für Strafgefangene ist rechtswidrig. Eine gesetzliche Einschränkung, d.h. eine rechtliche Grundlage für die Verweigerung eines solchen Anspruches existierte im StVollzG (Bund) und in den bereits verabschiedeten Ländergesetzen nicht. Lediglich der Musterentwurf hat sich des Problems angenommen. Die vollständige Verweigerung eines Internetzugangs kann auch nicht aus der Sorge um die Sicherheit und Ordnung der Anstalt hergeleitet werden. Darüber hinaus sind alle vermeintlichen Gefahren ebenfalls in anderen Kommunikations- und Informationsmitteln vorhanden, ohne dass der Zugang zu diesen völlig ausgeschlossen ist. Schließlich wirkt eine generelle Verweigerung eines Internetzugangs kontraproduktiv hinsichtlich des Ziels der Resozialisierung. 